\title{
Evaluation of a foot-to-foot bioelectrical impedance analyser in highly active, moderately active and less active young men
}

\author{
Ann M. Swartz*, M. Jeremy Evans, George A. King and Dixie L. Thompson \\ Department of Exercise Science and Sport Management, The University of Tennessee, Knoxville, TN, USA
}

(Received 10 August 2001 - Revised 4 March 2002 - Accepted 19 March 2002)

\begin{abstract}
The Tanita TBF-305 (Tanita Corp., Tokyo, Japan) is a commercially available foot-to-foot bioelectrical impedance analysis (BIA) system. The manufacturer-supplied equations incorporate gender, mass, height, activity category and a measured impedance value to determine $\%$ body fat (BF). The purpose of the present study was to determine whether the manufacturersupplied 'adult' and 'athlete' equations provided an accurate estimate of \% BF for a group of young men with varying activity levels. Fifty-seven men (18-35 years old) were categorized into the following groups: (1) highly active (HA) ( $\geq 10.0 \mathrm{~h}$ aerobic activity/week); (2) moderately active (MA) $(2 \cdot 5-10 \cdot 0 \mathrm{~h}$ aerobic activity/week); (3) less active (LA) $(<2.5 \mathrm{~h}$ aerobic activity/week). The \% BF was measured using the BIA 'athlete' and 'adult' modes. After BIA measurements, residual volume was measured and hydrostatic weighing (HW) was performed. The amount of activity performed by each group was significantly different $(P<0 \cdot 001)$. No significant differences were found between the $\% \mathrm{BF}$ determined by the 'athlete' mode and HW for HA $(P=0.309)$ and MA $(P=0.091)$. However, a significant difference was found for LA $(P=0.001)$. The $\%$ BF determined by the 'adult' mode and HW was not different for LA $(P=0.395)$, but was significantly different for MA $(P<0.001)$ and HA $(P<0.001)$. The choice of activity mode on the foot-to-foot BIA significantly alters prediction of $\% \mathrm{BF}$. With careful selection of activity mode, there was no statistical difference between $\%$ $\mathrm{BF}$ determined by $\mathrm{HW}$ and the BIA, but the range of individual error scores was large.
\end{abstract}

Body composition: Body fat: Exercise: Aerobic activity: Bioelectrical impedance analysis: Validation

Bioelectrical impedance analysis (BIA) has become a popular method of assessing body composition in settings ranging from research laboratories to individuals' homes. BIA systems measure the impedance when a current is applied to an individual's body. Generally, the impedance value, along with body height, body mass, and gender are incorporated into a mathematical equation, which results in estimates of $\%$ body fat (BF), fat-free mass (FFM) and total body water. Typically, the equations are populationspecific and are only appropriate for the group on which the equations were established. Scientific research has demonstrated that prediction of body composition with BIA at the extremes of body fatness are less accurate, with many equations overestimating fat mass in the lean, while underestimating fat mass in the obese (Segal et al. 1988). Therefore, it is very difficult to develop a BIA equation to predict $\% \mathrm{BF}$, FFM or total body water that will accurately serve a diversified population.
Individuals who engage in intense physical activity or are highly active (HA) tend to be leaner than their inactive or sedentary counterparts (Ballor \& Keesey, 1991; DiPietro, 1995; Ching et al. 1996). Thereby, BIA equations that are established on athletes may not predict accurately the body composition of inactive individuals and vice versa, BIA equations developed on inactive adults may not be accurate for athletes or HA individuals. In an attempt to increase accuracy, a single frequency, tetrapolar, foot-tofoot BIA system incorporated two preprogrammed body composition equations, one for moderately active (MA) to less active (LA) adults ('adult' mode) and one for HA adults ('athlete' mode). By incorporating an 'adult' and an 'athlete' equation in the same device, the system would theoretically be able to more accurately estimate the body composition of individuals spanning the spectrum of $\% \mathrm{BF}$.

The purpose of the present study was to test the accuracy

Abbreviations: BIA, bioelectrical impedance analysis; BF, body fat; FFM, fat-free mass; HA, highly active; HW, hydrostatic weighing; LA, less active; MA, moderately active.

* Corresponding author: Dr A. M. Swartz, present address, Dept of Physical Medicine and Rehabilitation, University of Michigan, 1500 E. Medical Center Drive, D4100 Medical Professional Building, Ann Arbor, MI 48109-0718, USA, fax +1 734936 7048, email annswar@med.umich.edu 
of the 'adult' and the 'athlete' equations supplied by the manufacturer of a commercially available BIA device for estimating \% BF and FFM in a group of young men with varying physical activity levels.

\section{Subjects and methods}

Fifty-seven Caucasian men between the ages of 18 and 35 years volunteered for the present study. All participants were recruited from the university and the surrounding community through public postings. Each participant was informed of potential risks and benefits, and signed an informed consent form approved by The University of Tennessee Institutional Review Board. All participants were categorized into the following groups: (1) HA men engaging in $\geq 10 \mathrm{~h}$ aerobic activity/week; (2) MA men engaging in $2 \cdot 5-10 \cdot 0 \mathrm{~h}$ aerobic activity/week; (3) LA men engaging in $<2.5 \mathrm{~h}$ aerobic activity/week. These groupings were chosen because the BIA manufacturer classifies an 'athlete' as one who engages in aerobic activity $\geq 10.0 \mathrm{~h} /$ week

Participants completed a series of body composition assessments including anthropometric measures, BIA assessment and hydrostatic weighing (HW). All measurements were performed in the morning after an overnight fast. In addition, participants were asked not to exercise for $12 \mathrm{~h}$ before the testing session, to abstain from alcohol for $48 \mathrm{~h}$ prior to testing, to consume water in their typical pattern and to empty their bladder immediately prior to testing. Finally, participants were excluded if they were taking any supplements or medications that would alter body water level.

\section{Anthropometric measures}

Standard anthropometric methods were used to determine body mass, body height, waist circumference, abdominal circumference and hip circumference (Harris, 1998). Body mass (in a Lycra, race-style swimsuit) and body height were measured to the nearest $0.1 \mathrm{~kg}$ and $0.001 \mathrm{~m}$ via a standard physician's scale (Health-o-meter, Bridgeview, IL, USA) and a stadiometer (Seca Corp., Columbia, MD, USA) respectively. BMI was calculated using the equation: body mass $(\mathrm{kg})$ divided by body height $(\mathrm{m})$ squared. Subjects with a BMI $>27.7 \mathrm{~kg} / \mathrm{m}^{2}$ were excluded. Waist, abdominal and hip circumferences were measured to the nearest $0.001 \mathrm{~m}$ using a measuring tape fitted with a tension spring. Waist:hip ratio was calculated by dividing the waist circumference by the hip circumference.

\section{Bioelectrical impedance analysis}

BIA measures were performed in accordance with the manufacturer's specified procedures. Participants were asked to remove all jewellery and other accessories. In addition, they were asked to remove all clothing except a swimsuit for the measurement. Gender, body height and physical activity classification were manually entered into the keypad interface. Body mass was automatically measured to the nearest $0.2 \mathrm{~kg}$ while simultaneous measurement of impedance was made. Body composition was assessed using both the 'athlete' mode and the 'adult' mode of the same BIA device.

The foot-to-foot, pressure contact electrode BIA system (Tanita TBF-305; Tanita Corp., Tokyo, Japan) uses two stainless-steel foot pad electrodes mounted on a platform scale. The scale consists of a single load cell that transforms mass placed on the scale into an electrical signal. The electrodes for each foot are subdivided into anterior and posterior electrodes. A current is applied through the anterior portion of the foot pad electrodes and the voltage drop is measured in the posterior portion of the foot pad electrodes. The impedance measurement uses a $50 \mathrm{kHz}-$ $500 \mu \mathrm{A}$ current and has an impedance range of $150-$ $900 \Omega$. The voltage drop and the body mass signal are converted to digital data using an analogue to digital converter. All BIA measures were made after at least $10 \mathrm{~min}$ standing to reduce possible errors from acute changes in body fluid distribution. Analysis of the impedance values from this device revealed a within day CV 0.6 (SD 0.6 ) $\%$ and a between-day CV 2.4 (SD 1.5) \%.

\section{Residual volume}

Residual volume was determined immediately after BIA measurement and immediately prior to the HW procedure. Residual volume was estimated using the modified $\mathrm{O}_{2}$ dilution procedure described by Wilmore (1969). Continuous gas analysis was performed with a Nitralyzer (KaeTech Instruments, Green Bay, WI, USA) equipped with a digital display. The residual volume measure was performed a minimum of two times to achieve two values within $100 \mathrm{ml}$. The average of these two trials was recorded as the residual volume.

\section{Hydrostatic weighing}

HW was performed using a submersion tank containing an electronic scale placed on four force cell transducers coupled to an integrated amplifier (Precision Biomedical Systems, Inc., University Park, PA, USA) (Akers \& Buskirk, 1969). The participant was asked to expel as much air as possible from his lungs and then submerge himself underwater. The procedure was repeated six to ten times. Underwater mass was recorded as the average of the three highest documented mass measures within $0 \cdot 1 \mathrm{~kg}$. Body density was determined from underwater mass using the equation of Goldman \& Buskirk (1961). Gastrointestinal gases were assumed to be $100 \mathrm{ml}$. The \% $\mathrm{BF}$ was calculated from body density using the equation of Siri (1961). The CV for \% BF measurements was $3.0 \%$.

\section{Statistical methods}

Overall mean subject characteristics were compared between groups using the ANOVA procedure. Post-hoc testing with Bonferonni adjustment was carried out to determine between group differences. The impedance scores between the 'athlete' and 'adult' modes of the BIA system were compared with a paired $t$ test. Overall mean \% BF and FFM scores were compared using twoway repeated-measures ANOVA. Post-hoc testing with 
Table 1. Descriptive characteristics of participants

(Mean values and standard deviations)

\begin{tabular}{|c|c|c|c|c|c|c|}
\hline & \multicolumn{2}{|c|}{$\begin{array}{l}\text { Highly active } \\
\qquad(n 17)\end{array}$} & \multicolumn{2}{|c|}{$\begin{array}{c}\text { Moderately } \\
\text { active }(n 20)\end{array}$} & \multicolumn{2}{|c|}{$\begin{array}{l}\text { Less active } \\
\quad(n 20)\end{array}$} \\
\hline & Mean & SD & Mean & SD & Mean & SD \\
\hline Age (years) & $23 \cdot 6$ & $4 \cdot 8$ & $24 \cdot 0$ & 4.4 & $22 \cdot 2$ & $3 \cdot 1$ \\
\hline Height (m) & 1.81 & 0.05 & $1 \cdot 78$ & 0.04 & 1.82 & 0.08 \\
\hline Body mass $(\mathrm{kg})$ & 78.6 & 9.2 & 74.6 & $6 \cdot 2$ & $79 \cdot 3$ & 9.7 \\
\hline $\operatorname{BMI}\left(\mathrm{kg} / \mathrm{m}^{2}\right)$ & 23.9 & $2 \cdot 0$ & 23.5 & $2 \cdot 1$ & $23 \cdot 8$ & $2 \cdot 3$ \\
\hline Waist:hip ratio & 0.81 & 0.04 & 0.79 & 0.04 & 0.81 & 0.03 \\
\hline Aerobic activity (h/week) & $14 \cdot 2^{* \star *}$ & 1.7 & $5 \cdot 0^{\star \star *}$ & $2 \cdot 1$ & $1 \cdot 0^{* \star *}$ & 0.6 \\
\hline
\end{tabular}

Mean values were significantly different from each other: ${ }^{\star \star \star} P<0.001$.

Bonferroni adjustment was performed where appropriate to locate significant differences between groups. BlandAltman (Bland \& Altman, 1986) plots were created to examine the systematic differences in the methods for each group. Pearson product-moment correlation $(r)$ was used to determine the relationship between the \% $\mathrm{BF}$ error scores and the average of the $\% \mathrm{BF}$ values for each group, between quantity of physical activity performed and $\% \mathrm{BF}$ error scores, and quantity of physical activity performed and \% BF by HW for all participants. All data were analysed using SPSS for Windows, version 10.0.0 (SPSS Inc., Chicago, IL, USA). Significance level was set at $P \leq 0 \cdot 05$.

\section{Results}

The characteristics of the study participants are displayed in Table 1. There were no significant differences between the HA, MA or LA groups for age, height, body mass, BMI or waist:hip ratio. All groups significantly differed from each other in the $\mathrm{h}$ aerobic activity/week $(P<0 \cdot 001)$.

The \% BF (Table 2) determined by HW was not significantly different compared with the BIA 'athlete' mode for the HA $(P=0.309)$ and MA $(P=0.091)$ groups. However, a significant difference was found between HW and the BIA 'athlete' mode for the LA group $(P=0 \cdot 001)$. The \% BF determined by $\mathrm{HW}$ and the 'adult' mode was not significantly different for the LA group $(P=0 \cdot 395)$, but was significantly different for the HA $(P<0.001)$ and MA $(P<0.001)$ groups. The association between the estimation techniques and activity groups for FFM values was similar to those for $\% \mathrm{BF}$ (Table 2). It is important to note that although there were differences between the BIA 'athlete' and 'adult' modes for $\% \mathrm{BF}$ and FFM, a paired samples $t$ test revealed there was no significant difference $(P=0 \cdot 147)$ between the impedance values measured in the 'athlete' mode $v$. those measured in the 'adult' mode (500.7 $v$. $500 \cdot 2 \Omega$ ) for all participants.

Fig. 1 shows the individual differences between HW and BIA for both the 'athlete' and 'adult' modes for the HA, MA and LA groups, as well as the Pearson product moment correlations for these relationships. An examination of these figures shows that the 'athlete' mode for the HA (Fig. 1(a)) and MA (Fig. 1(c)) groups and the 'adult' mode for the LA group (Fig. 1(f)) had a mean error of approximately $2 \%(1.8,2.1$ and $-1.8 \%$ for HA, MA and LA respectively) and limits of agreement ranging from approximately \pm 8 to $\pm 10 \%$ ( $\pm 8.66, \pm 8.00$, $\pm 10.42 \%$ for HA, MA and LA respectively). The use of the 'adult' mode in HA (Fig. 1(b)) and MA (Fig. 1(d))

Table 2. Percentage body fat and fat-free mass estimates from hydrostatic weighing, body impedance analysis 'athlete' and body impedance analysis $¥ \S$ 'adult' modes (Mean values with their standard errors)

\begin{tabular}{|c|c|c|c|c|c|c|c|}
\hline & \multirow[b]{2}{*}{$n$} & \multicolumn{2}{|c|}{ HW } & \multicolumn{2}{|c|}{$\begin{array}{l}\text { BIA 'athlete' } \\
\text { mode }\end{array}$} & \multicolumn{2}{|c|}{ BIA 'adult' mode } \\
\hline & & Mean & SE & Mean & SE & Mean & SE \\
\hline \multicolumn{8}{|l|}{ Body fat (\%) } \\
\hline Highly active & 17 & $12 \cdot 5$ & $1 \cdot 2$ & $10 \cdot 7$ & 0.7 & $17 \cdot 5^{\star \star \star}+\dagger \dagger$ & 0.9 \\
\hline Moderately active & 20 & $12 \cdot 1$ & $1 \cdot 2$ & $10 \cdot 0$ & 0.6 & $16 \cdot 8^{\star * *}+\dagger \dagger$ & $0 \cdot 8$ \\
\hline Less active & 20 & $16 \cdot 4$ & $1 \cdot 2$ & $11 \cdot 9^{\star \star \star}$ & 0.8 & 18.2††† & $1 \cdot 0$ \\
\hline \multicolumn{8}{|l|}{ Fat-free mass $(\mathrm{kg})$} \\
\hline Highly active & 17 & $68 \cdot 5$ & 1.5 & $70 \cdot 0$ & $1 \cdot 7$ & $64 \cdot 5^{\star \star \star}+† \dagger$ & 1.3 \\
\hline Moderately active & 20 & $65 \cdot 4$ & $1 \cdot 0$ & $67 \cdot 1$ & $1 \cdot 0$ & 61.9** ††† & 0.8 \\
\hline Less active & 20 & $66 \cdot 2$ & $1 \cdot 8$ & $69 \cdot 9^{\star \star \star}$ & $1 \cdot 8$ & 64.6††† & 1.5 \\
\hline
\end{tabular}

$\mathrm{HW}$, hydrostatic weighing; BIA, bioelectrical impedance analysis.

Mean values were significantly different from those for $\mathrm{HW}:{ }^{\star \star \star} P<0.001$.

Mean values were significantly different from those for BIA 'athlete' mode: $\uparrow \dagger \uparrow P<0.001$.

‡For details of subjects and procedures, see Table 1 and p. 206.

$\S$ Tanita TBF-305; Tanita Corp., Tokyo Japan. 

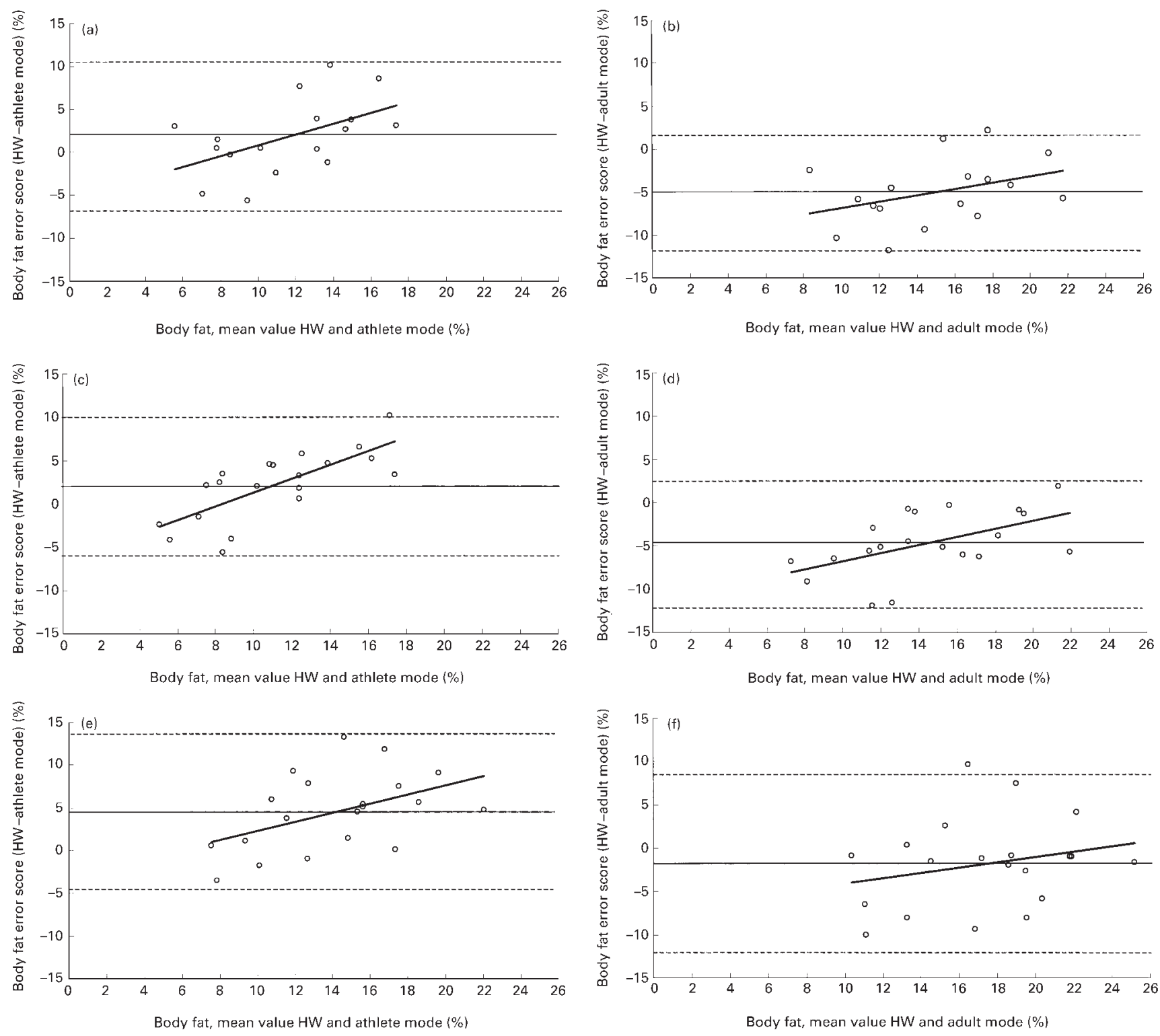

Fig. 1. Bland-Altman plots to determine systematic differences in \% body fat for hydrostatic weighing (HW) and bioelectrical impedance analyser (Tanita TBF-305; Tanita Corp., Tokyo, Japan) 'athlete' mode, and hydrostatic weighing and bioelectrical impedance analyser 'adult' mode for the highly active ((a) and (b) respectively) moderately active ((c) and (d) respectively), and less active ((e) and (f) respectively) groups. For details of subjects and procedures, see Table 1 and p. 206. - Mean difference; ----, \pm 2 SD. (a), $r 0.513, P=0.035 ;(b), r 0.0379, P=0.134$; (c), $r 0.744, P<0.001 ;$ (d), $r 0.526, P=0.017$; (e), $r 0.468, P=0.038$; (f), $r 0.244, P<0.300$.

men and the 'athlete' mode in LA men (Fig. 1(e)) resulted in a mean error of at least $4.5 \%(5 \cdot 0,4.7$ and $4.5 \%$ for HA, MA and LA respectively) and limits of agreement ranging from approximately \pm 7 to $\pm 9 \%( \pm 7.67, \pm 7.32$ and $\pm 9.03 \%$ for HA, MA and LA respectively).

When examining the quantity of physical activity performed as a continuous variable, the data shows that the relationship between the \% $\mathrm{BF}$ error scores and amount of physical activity performed per week was not significant ('athlete', $\quad r-0.217, \quad P=0.105$; 'adult', $\quad r-0.232$, $P=0.082$; Fig. 2(a and b) respectively). There was a small but significant relationship between $\%$ BF by HW and quantity of physical activity performed per week $(r$ $0.273, \quad P=0.04)$, where amount of physical activity accounted for $7.5 \%$ of the variance in body fat.

\section{Discussion}

The need for accurate methods of assessing body composition has been amplified in recent years with the growing interest in athletic performance, as well as the increasing rates of obesity and obesity-related disorders. The ease of use and immediate feedback provided by a portable footto-foot BIA makes it an attractive choice for $\% \mathrm{BF}$ estimates in research settings, health-care facilities and individuals' homes. Studies have shown the foot-to-foot BIA device to be a useful alternative to the conventional, more laborious and technical tetrapolar BIA systems (Nunez et al. 1997; Jebb et al. 2000). Although investigators have evaluated the accuracy of select foot-to-foot systems (Utter et al. 1999; Franckowiak et al. 2000), no 

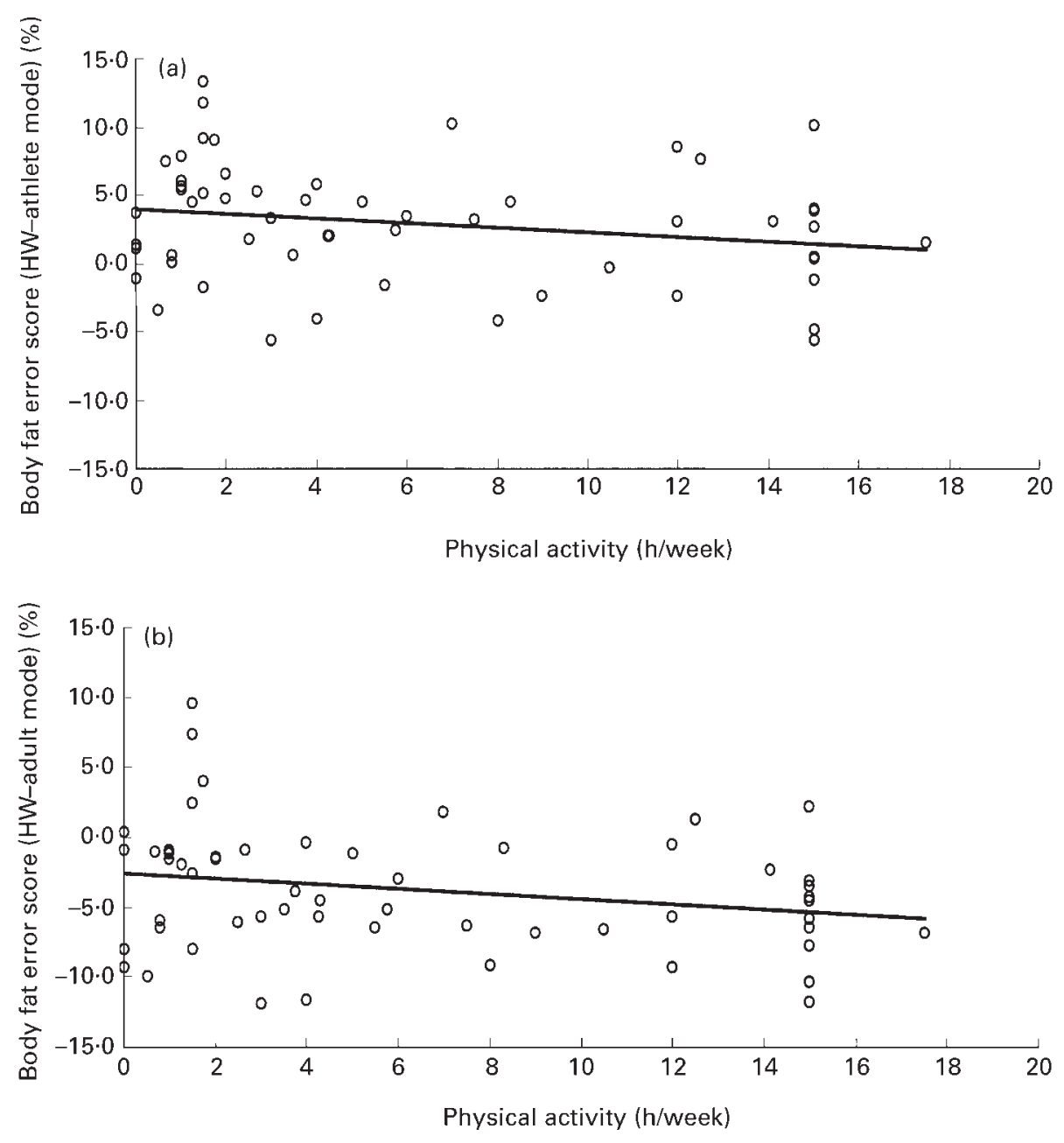

Fig. 2. Physical activity performed (h/week) and \% body fat error scores for: (a), hydrostatic weighing (HW) minus 'athlete' mode of the bioelectrical impedance analyser (Tanita TBF-305; Tanita Corp., Tokyo, Japan); (b), HW minus 'adult' mode of bioelectrical impedance analyser. For details of subjects and procedures, see Table 1 and p. 206. (a), $r-0.217, P=0.105 ;(b), r-0.232, P=0.082$.

current data exists on the accuracy of the BIA foot-to-foot systems that account for physical activity level. The importance of incorporating physical activity levels into body composition estimates stems from research showing that BIA assessment of body composition was less accurate for individuals with very high BF levels and individuals with very low BF levels compared with individuals with a moderate level of BF (Segal et al. 1988). Because HA individuals have a tendency to be leaner than their inactive or sedentary counterparts (Ballor \& Keesey, 1991; DiPietro, 1995; Ching et al. 1996), a single BIA equation may not work as well for HA individuals or athletes as it does for MA or LA individuals. Therefore, the intent of the present study was to evaluate a foot-to-foot BIA system that incorporates two pre-programmed body composition equations, one for MA to LA adults ('adult' mode), and one for the HA adult ('athlete' mode).

Results from our current study show that \% BF determined by the 'athlete' mode of the BIA machine was not significantly different from HW for men who performed at least $2.5 \mathrm{~h}$ aerobic activity/week (Table 2). However, for HA and MA men who were at the higher end of the
$\% \mathrm{BF}$ range, the 'athlete' mode was more likely to under-predict their \% BF (Fig. 1(a and c) respectively). The BIA 'adult' mode accurately assessed \% BF compared with $\mathrm{HW}$ for men who engage in $<2.5 \mathrm{~h}$ aerobic activity/ week (Table 2). Taken together, the results from the HA and LA groups correspond with the manufacturer's instructions pertaining to physical activity level and mode choice. For MA men, the 'athlete' mode was most appropriate, even though manufacturer's recommendations would label them in the 'adult' category. However, for all groups, individual errors were still relatively large (Fig. 1), ranging from \pm 8.0 to $\pm 10.4 \%$. This indicates that although group means were not significantly different when mode selection was appropriate, on an individual level, sizeable differences between HW and BIA estimates of $\% \mathrm{BF}$ were apparent.

It is important to note that results from the current investigation showed that there was no difference in the impedance values measured by the 'athlete' mode and the 'adult' mode (500.7 v. 500.2 $\Omega$ respectively). Therefore, the difference between the $\% \mathrm{BF}$ and FFM values estimated from the different test modes is based on the 
pre-programmed regression equation supplied by the manufacturer and not the impedance values determined by the BIA machine.

Although there was not a statistically significant correlation between exercise (h/week) and the accuracy of prediction for either BIA mode (Fig. 2), there was a trend for the BIA predictions to be higher as activity levels were higher. This resulted in prediction by the 'athlete' mode being more accurate (i.e. mean differences closer to zero) for the HA men (Fig. 2(a)). In addition, the predictions by the 'adult' mode were more accurate for men engaging in little to no exercise (Fig. 2(b)). The rather narrow range of $\% \mathrm{BF}$ for our subjects may have lowered the correlations reported in Fig. 2. With a more heterogeneous group of men, the influence of exercise in predicting \% BF may be even greater.

A potential limitation of the current study is the use of HW and the two-compartment model as the criterion measure for body composition. HW is a suitable criterion method in the present study for the following reasons. HW has been shown to be a valid and reliable measure of body composition with good test-retest reliability ( $r$ 0.99) (Ward et al. 1978), and good agreement with a four-compartment estimate of fat mass ( $r$ 0.986) (Jebb et al. 2000). In addition, the young men used within the current study were similar to those men used to develop the Siri (1961) equation. Another limitation is that the study population consisted of a fairly small homogeneous group of non-obese, young men. Therefore, the results are only applicable to a population with similar attributes, and further study needs to occur to fully explore the applicability of this device.

\section{Conclusion}

In summary, the present study found that the 'adult' mode accurately reflected group \% BF estimates in individuals performing $<2.5 \mathrm{~h}$ aerobic activity/week, and that the 'athlete' mode accurately estimated group \% BF estimates of individuals engaging in $\geq 2.5 \mathrm{~h}$ aerobic activity/week; therefore, choice of mode is critically important when using this device. In addition, individual errors were sometimes quite large, making this device unacceptable for 'criterion' estimates of body composition. Additional studies are required that incorporate women, older adults and different ethnic groups to establish fully the usefulness of available regression formulas for differing physical activity levels utilizing foot-to-foot BIA technology.

\section{Acknowledgements}

The authors would like to thank Cary Springer for guidance with the statistical analysis in this paper and Scott Strath, Jody Clasey and Edward T. Howley for thoughtful comments in preparing this manuscript.

\section{References}

Akers R \& Buskirk E (1969) An underwater weighing system utilizing "force cube" transducers. Journal of Applied Physiology 26, 649-652.

Ballor D \& Keesey R (1991) A meta-analysis of the factors affecting exercise-induced changes in body mass, fat mass and fat-free mass in males and females. International Journal of Obesity 15, 717-726.

Bland J \& Altman D (1986) Statistical methods for assessing agreement between two methods of clinical measurement. Lancet 8, 307-310.

Ching P, Willett W, Rimm E, Colditz G, Gortmaker S \& Stampfer MJ (1996) Activity level and risk of overweight in male health professionals. American Journal of Public Health 86, 25-30.

DiPietro L (1995) Physical activity, body weight, and adiposity: an epidemiologic perspective. Exercise and Sport Science Reviews 23, 275-303.

Franckowiak S, Cotton R, Ritter M, Walston J, Beamer B, Fritsch L \& Andersen R (2000) Accuracy of a low-cost, commercially available Tanita bioelectrical impedance analyzer to estimate body composition. Medicine and Science in Sports and Exercise 32, $113 \mathrm{~S}$.

Goldman R \& Buskirk E (1961) Body volume measurement by underwater weighing: Description of method. In Techniques for Measuring Body Composition, pp. 78-89 [J Brozek and A Henschel, editors]. Washington, DC: National Academy of Sciences-National Research Council.

Harris G (1998) Skinfold thickness and measurement technique. In Anthropometric Standardization Reference Manual, pp. 55-70 [T Lohman, A Roche and R Martorell, editors]. Champaign, IL: Human Kinetics Publishers.

Jebb SA, Cole TJ, Doman D, Murgatroyd PR \& Prentice AM (2000) Evaluation of the novel Tanita body-fat analyser to measure body composition by comparison with a four-compartment model. British Journal of Nutrition 83, 115-122.

Nunez C, Gallagher D, Visser M, Pi-Sunyer FX, Wang Z \& Heymsfield SB (1997) Bioimpedance analysis: evaluation of leg-to-leg system based on pressure contact foot-pad electrodes. Medicine and Science in Sports and Exercise 29, $524-531$.

Segal K, Van Loan M, Fitzgerald P, Hodgdon J \& Van Itallie T (1988) Lean body mass estimation by bioelectrical impedance analysis: a four-site cross-validation study. American Journal of Clinical Nutrition 47, 7-14.

Siri W (1961) Body composition from fluid spaces and density: Analysis of methods. In Techniques for Measuring Body Composition, pp. 223-224 [J Brozek and A Henschel, editors]. Washington, DC: National Academy of Sciences-National Research Council.

Utter A, Nieman D, Ward A \& Butterworth D (1999) Use of the leg-to-leg bioelectrical impedance method in assessing bodycomposition change in obese women. American Journal of Clinical Nutrition 69, 603-607.

Ward A, Pollock M, Jackson A, Ayres J \& Pape G (1978) A comparison of body fat determined by underwater weighing and volume displacement. American Journal of Physiology 234, E94-E96.

Wilmore J (1969) A simplified method for determination of residual lung volume. Journal of Applied Physiology 27, 96-100. 